\title{
Capítulo 23
}

\section{Análise paramétrica de vigas de concreto com fibras metálicas}

Wanderlei Malaquias

José Júlio de Cerqueira Pituba ${ }^{2}$

Pereira Junior ${ }^{1}$

Resumo: Este trabalho trata de uma abordagem numérica da aplicação do modelo constitutivo de mecânica do dano na análise do comportamento estrutural do concreto armado reforçado com fibras metálicas. Essa abordagem ainda é nova visto que o Brasil não possui nenhuma normatização em relação ao procedimento de cálculo desse tipo de material. O presente estudo expõe como objetivo fundamental a análise paramétrica das vigas estudadas por Lopes (2005) e Pereira Junior (2014). Nesse trabalho, variações de altura $(b)$, largura $\left(b_{w}\right)$, área de aço $(A s)$ e vão $(L)$ são empregadas de forma a verificar a influência de cada parâmetro no estudo numérico da deformabilidade de peças fissuradas compostas por este material. Observa-se que os resultados obtidos com o emprego de modelagem a dano refletem o comportamento real das estruturas analisadas, onde parâmetros de maior influência são destacados.

Palavras-chave: Mecânica do dano, Concreto com fibra, Vigas, Modelos constitutivos.

\section{Introdução}

Modelos constitutivos têm fundamental importância na Engenharia de Estruturas visto que os mesmos auxiliam na determinação do comportamento mecânico de sistemas mecânicos em geral. Pereira Junior (2014) descreve uma

1 Universidade Federal de Goiás - UFG. Regional Catalão, Unidade Acadêmica Especial de Engenharias. Laboratório de Mecânica Computacional. Contato: wanderlei.malaquias. pereira.jr@gmail.com.

2 Universidade Federal de Goiás - UFG. Regional Catalão, Unidade Acadêmica Especial de Engenharias. Laboratório de Mecânica Computacional. Contato: Julio.pituba@pq.cnpq. br. Bolsista de Produtividade em Pesquisa do CNPq. 
série de modelos utilizados para a análise de estruturas, como os modelos de plasticidade, fratura e dano, sendo o último, foco desse trabalho.

$\mathrm{Na}$ Engenharia Civil, três materiais se destacam e são amplamente utilizados como material estrutural, sendo: aço; madeira; concreto. Porém, no Brasil, o concreto tem um destaque maior entre os três tipos, sendo que desde a década de 1950, um dos mais utilizados como material estrutural em construção de pequeno a grande porte.

De maneira geral, entender o comportamento mecânico do concreto não é uma tarefa simples, então modelos como os citados acima são importantes para o entendimento desse comportamento mecânico visto que ensaios tem um alto custo financeiro, além do que a simulação numérica auxilia na calibração de modelos reais, permitindo uma maior amplitude de testes quando comparada ao processo experimental.

O trabalho tem como objetivo tratar do estudo paramétrico de estruturas de vigas com concreto armado reforçado com fibras metálicas verificando assim a deformabilidade de cada variação estudada. Como o modelo constitutivo utilizado foi baseado na Mecânica do Dano, verificou-se então o deslocamento das peças utilizando esse tipo de modelo constitutivo.

Esse trabalho justifica-se devido à necessidade do melhor entendimento do comportamento de estruturas quando executadas em concreto armado e reforçadas com fibras metálicas. Outro fator importante é o fato de que o Brasil ainda não tem nenhum procedimento para verificação no Estado Limite Último (ELU) e Estado Limite de Serviço (ELS) para peças de concreto reforçada com fibras metálicas.

\section{Desenvolvimento}

No item 1 é apresentando o material estrutural, concreto com fibras, utilizado nas simulações. Logo após, toda a formulação matemática do modelo de dano e homogeneização dos materiais é discutida.

\subsection{Concreto com fibras metálicas}

Segundo Scoaris (2005), a fibra de aço é usada principalmente para controle da fissuração e, em determinados casos, a armadura principal pode ser substituída pela mesma, por exemplo, em pisos de concreto. Este material pode ser utilizado em lajes de edifícios, pavimentos e túneis, como também em recuperações de estruturas.

A fibra de aço quando misturada ao concreto simples se comporta como uma barreira para a propagação da fissuração ao longo da peça de concreto. Todavia a 
capacidade de impedir estes efeitos de tração causados pela fissuração é principalmente determinada pela interação entre a matriz cimentícia e a fibra em questão, tornando então o estudo do comportamento mecânico entre a fibra e a matriz cimentícia de grande importância na determinação das propriedades mecânicas finais do concreto (PEREIRA JUNIOR, 2014)

Portanto, a fibra metálica tem a capacidade de promover o grampeamento das fissuras reduzindo assim a concentração de tensões na ponta da trinca. Esse efeito mitiga o processo de fissuração por completo na peça aumentando assim a capacidade portante da peça, visto que a mesma terá um comportamento mais dúctil.

Pasa (2007) cita alguns fatores que interferem no comportamento da fibra metálica na matriz cimentícia, são elas:

- A condição da matriz, antes ou após a fissuração;

- Propriedades mecânicas da matriz e da fibra;

- Geometria da fibra (comprimento, forma, diâmetro e mecanismos de ancoragem);

- Teor de fibras no compósito.

O Brasil ainda não possui nenhum processo normativo que regulamenta o dimensionamento de peças de concreto armado com fibras metálicas. O único procedimento normativo relativo a esse assunto foi criado recentemente, porém com o intuito apenas de classificar as fibras metálicas pela sua tipologia. Portanto, somente a NBR 15530 (ABNT, 2007) trata de forma mais direta da fibra metálica em misturas de concreto. Existe também outro procedimento normativo que cita o uso de fibras metálicas que é a norma NBR 8890 (ABNT, 2007), a qual prevê o uso de fibras metálicas na fabricação de tubos ou manilhas de concreto para águas pluviais.

\subsection{Descrição dos modelos computacionais utilizados}

Os modelos computacionais são descritos nos itens 1.2.1 ao item 1.2.3, tendo como base a formulação desenvolvida em Pituba e Fernandes (2011), Pereira Junior (2014) e Pereira Junior et al. (2016), onde maiores detalhes podem ser encontrados.

\subsubsection{Modelo das armaduras}

Neste trabalho foi utilizado um modelo constitutivo unidimensional simulando o comportamento mecânico das armaduras longitudinais no concreto armado. Sendo que o modelo aqui utilizado é um modelo de diagrama bilinear para representação do comportamento tensão versus deformação desse material. 


\subsubsection{Modelo de dano para concreto}

O modelo utilizado para descrever o comportamento do concreto é baseado na mecânica do dano. Esse modelo é embasado na análise da microfissuração do material, sendo que a variável de dano é representada por um escalar $D$ que penaliza o módulo de elasticidade $E$ do material. No caso desse modelo desenvolvido por Pituba e Fernandes (2011) foi adotado apenas a sua versão unidimensional, visto que esta é mais simplificada e pode ser utilizada como um critério para análise do comportamento carga versus deslocamento, apresentando bons resultados como descrito em Pituba (2003) e Pereira Junior (2014).

O modelo de dano simula o comportamento de uma peça em processo de fissuração através de um sólido deformado equivalente, chamado de sólido de volume representativo, para que as funções que representam o comportamento do mesmo sejam continuas. Para tal processo o valor do escalar de dano é dado pela equação (1.1).

$$
D_{n}=\lim _{S \rightarrow 0} \frac{S_{0}}{S}
$$

Onde:

$S_{0}$ - Área de defeitos ou microfissuras;

$S$ - Área total da seção;

A área que efetivamente resiste aos esforços ou simplesmente $S_{d}$ é definida pela equação (1.2), apresentada logo abaixo.

$$
S_{d}=S-S_{0}
$$

Considerando o princípio da equivalência de energia para verificação do sólido equivalente chega-se a equação (1.3) que representa o escalar de dano considerando os valores dos módulos de elasticidade antes e após a penalização causada pela danificação.

$$
D=1-\left(\frac{E_{\theta}}{E}\right)^{1 / 2}
$$

Onde:

$E_{\theta}$ - Módulo de elasticidade efetiva;

$E$ - Módulo de elasticidade inicial;

O modelo de dano em estudo está implementado em linguagem Fortran em um código computacional baseado no Método dos Elementos Finitos para a análise numérica de estruturas em barras discretizadas com seção transversal estratificada (ver Figura 23.1), permitindo assim considerar na seção transversal camadas 
equivalentes de aço ou concreto. Tal código é identificado aqui como EFICOS. Ao longo deste trabalho admite-se perfeita aderência entre concreto e aço, definindo assim para cada camada suas características, como módulo de elasticidade e deformações anelásticas equivalentes, utilizando regra de homogeneização para tal fim (PEREIRA JUNIOR ,2014).

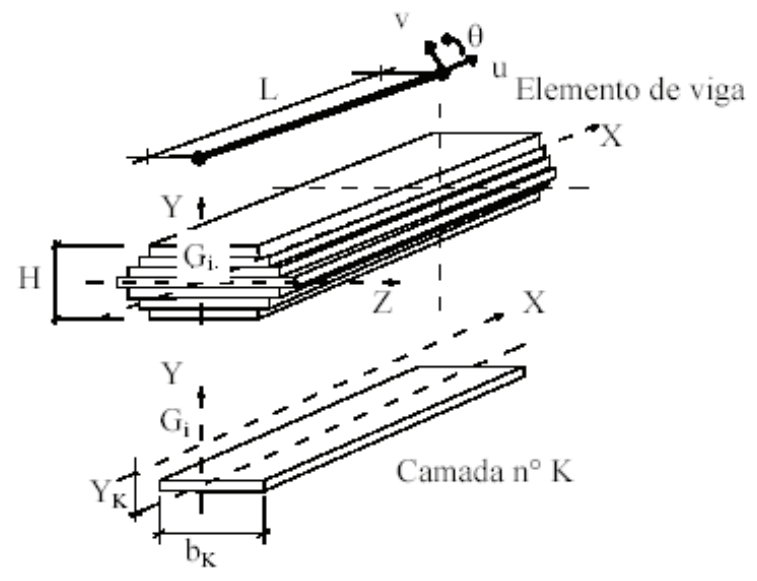

Figura 23.1 Elemento finito empregado no código computacional (PITUBA, 2003)

Para o modelo de elementos finitos, o valor do dano é dado pela equação (1.4), representando assim o valor do dano escalar.

$$
D_{i}=1-\frac{1+A_{i}}{A_{i}+e^{\left[B_{i}\left(Y_{i}-Y_{\mathrm{oi}}\right)\right]}}
$$

Os parâmetros $A, B$ e $Y$ estão relacionados com o comportamento da curva tensão versus deformação do sólido. Em Pereira Junior (2014) foi realizada uma simulação para verificar a influência de cada parâmetro no comportamento da peça.

O sub-índice $i$ denota se a variável de dano indica uma danificação por tração ou compressão. Maiores detalhes sobre a formulação encontram-se em Pituba e Fernandes (2011) e Pereira Junior (2014).

\subsubsection{Modelo de homogeneização para consideração da presença das fibras no concreto}

O comportamento mecânico de estruturas de concreto reforçado com fibras, quando submetidas a situações de carregamento e descarregamento, depende das interações da matriz cimentícia. Neste artigo, o procedimento utilizado para consideração das interações entre fibra/matriz é o procedimento de La Borderie (1991). 
Por outro lado, via método de homogeneização cinemática de Voigt, La Borderie (1991) propõe a expressão (1.5) para o cálculo da tensão homogeneizada do compósito de concreto reforçado com fibras metálicas:

$$
\sigma_{C R F A}=(1-C) \sigma_{m}+C \sigma_{f}
$$

Onde:

$\sigma_{C R F A}$ : Tensão do material homogeneizado, dito CRFA;

$C$ : Fração volumétrica de fibras de aço;

$\sigma_{m}:$ Tensão na matriz cimenticia;

$\sigma_{f}$ : Tensão na interface fibra tracionada imersa no concreto.

Observa-se que foi introduzida uma hipótese simplificadora, onde a deformação é suposta idêntica para a matriz e a fibra. Além disso, a orientação das fibras, que é aleatória e não privilegiada no sentido da solicitação, não é levada em conta na modelagem proposta (PEREIRA JUNIOR, 2014).

\section{Metodologia / procedimentos utilizados}

O procedimento desse trabalho baseia-se em testes numéricos de vigas, onde o foco é a análise carga versus deslocamento em vigas de seção retangular. $\mathrm{O}$ método utilizado consiste em variar dimensões da peça como, altura, largura e vão e também a área de aço da seção transversal da viga.

O modelo inicial, denominado aqui de s0, é o mesmo do trabalho experimental de Lopes (2005) e os dados iniciais do modelo constitutivo de dano e fibras metálicas são os mesmos obtidos por Pereira Junior (2014) para a viga com concentração de 2,00\% de fibras metálicas. As Tabelas 2.1 e 2.2 apresentam os valores utilizados para o modelo de dano e para as variáveis que consideram as fibras no problema, respectivamente.

Tabela 23.1 Variáveis de dano para os testes numéricos (PEREIRA JUNIOR, 2014)

\begin{tabular}{ccccccccc}
\hline $\begin{array}{c}\text { Módulo de } \\
\text { Elasticidade } \\
(\mathrm{MPa})\end{array}$ & At & Ac & Bt & Bc & YOt & Yoc & Betat & Betac \\
\hline 30190,00 & 15,00 & 0,70 & 1290 & 2,5 & $9 \mathrm{E}-05$ & 0,005 & $5 \mathrm{E}-07$ & $3 \mathrm{E}-04$ \\
\hline
\end{tabular}

Tabela 23.2 Variáveis da fibra metálica para os testes numéricos (PEREIRA JUNIOR, 2014)

\begin{tabular}{cccc}
\hline $\begin{array}{c}\text { Tensão de } \\
\text { Pico (MPa) }\end{array}$ & $\begin{array}{c}\text { Tensão de } \\
\text { escoamento (MPa) }\end{array}$ & $\begin{array}{c}\text { Deformação de } \\
\text { Ruptura (\%) }\end{array}$ & $\begin{array}{c}\text { Concentração } \\
\text { de fibras (\%) }\end{array}$ \\
\hline 525,00 & 420,00 & 2,50 & 2,00 \\
\hline
\end{tabular}


Essas variáveis são mantidas fixas durante os testes visto que as variáveis de dano e das fibras são obtidas através de ensaios experimentais do material. Esses ensaios são descritos com mais detalhes em Pituba e Fernandes (2011) e Pereira Junior (2014).

Neste trabalho, como descrito anteriormente, apenas alguns parâmetros da peça foram alterados, e para descrever melhor esses testes as Tabelas 2.3 a 2.6 são apresentadas.

As duas primeiras Tabelas (ver Tabelas 2.3 e 2.4) são referentes a mudanças na seção transversal do elemento, primeiramente mudanças na altura e depois mudanças na largura da peça de viga. Para efeito de testes, ou $b$ ou $h$ foram mantidas constantes ao longo desses dois testes iniciais, dependendo do que era variável.

Tabela 23.3 Teste 1 realizado para mudança de altura

\begin{tabular}{cllccl}
\hline Situações & $h(\mathrm{~cm})$ & $b(\mathrm{~cm})$ & $L(\mathrm{~cm})$ & $A s(\mathrm{~cm} 2)$ & $h / b$ \\
\hline 0 & 25,0000 & 12,5000 & 230,0000 & 4,0220 & 2,00 \\
1 & 26,2500 & 12,5000 & 230,0000 & 4,0220 & 2,10 \\
2 & 28,1250 & 12,5000 & 230,0000 & 4,0220 & 2,25 \\
3 & 31,2500 & 12,5000 & 230,0000 & 4,0220 & 2,50 \\
4 & 34,3750 & 12,5000 & 230,0000 & 4,0220 & 2,75 \\
5 & 37,5000 & 12,5000 & 230,0000 & 4,0220 & 3,00 \\
\hline
\end{tabular}

Tabela 23.4 Teste 2 realizado para mudança de largura

\begin{tabular}{cccccc}
\hline Situações & $h(\mathrm{~cm})$ & $b(\mathrm{~cm})$ & $L(\mathrm{~cm})$ & $A s(\mathrm{~cm} 2)$ & $b / h$ \\
\hline 0 & 25,0000 & 12,50 & 230,0000 & 4,0220 & 0,50 \\
1 & 25,0000 & 52,50 & 230,0000 & 4,0220 & 2,10 \\
2 & 25,0000 & 56,25 & 230,0000 & 4,0220 & 2,25 \\
3 & 25,0000 & 62,50 & 230,0000 & 4,0220 & 2,50 \\
4 & 25,0000 & 68,75 & 230,0000 & 4,0220 & 2,75 \\
5 & 25,0000 & 75,00 & 230,0000 & 4,0220 & 3,00 \\
\hline
\end{tabular}

As reduções de armadura simulam valores de perda de $10 \%$ a $50 \%$ do valor inicial dado no teste 0 que é de $4,02 \mathrm{~cm}^{2}$ de aço.

Tabela 23.5 Teste 3 realizado para mudança da área de aço

\begin{tabular}{cccccc}
\hline Situações & $h(\mathrm{~cm})$ & $b(\mathrm{~cm})$ & $L(\mathrm{~cm})$ & As $(\mathrm{cm} 2)$ & $\begin{array}{c}\text { redução armadura } \\
\%\end{array}$ \\
\hline 0 & 25,0000 & 12,50 & 230,0000 & 4,02 & --- \\
1 & 25,0000 & 12,50 & 230,0000 & 3,62 & 10,00 \\
2 & 25,0000 & 12,50 & 230,0000 & 3,22 & 20,00 \\
3 & 25,0000 & 12,50 & 230,0000 & 2,82 & 30,00 \\
4 & 25,0000 & 12,50 & 230,0000 & 2,41 & 40,00 \\
5 & 25,0000 & 12,50 & 230,0000 & 2,01 & 50,00 \\
\hline
\end{tabular}


O teste 4 é realizado com uma mudança no vão da peça. É importante ressaltar que nessa simulação a distância entre as cargas da flexão a 4 pontos foi sempre mantida em uma faixa próxima de L/3, garantindo assim que o teste tenha sempre o mesmo padrão dos anteriores permitindo que a comparação da carga versus deslocamento seja feita de maneira adequada. Toda a área de aço é mantida constante em $A s=4,02 \mathrm{~cm}^{2}$.

Tabela 23.6 Teste 4 realizado para mudança do vão

\begin{tabular}{ccccccc}
\hline Situações & $\mathrm{h}(\mathrm{cm})$ & $\mathrm{b}(\mathrm{cm})$ & $\mathrm{L}(\mathrm{cm})$ & $\begin{array}{c}\text { vão livre } \\
(\mathrm{cm})\end{array}$ & $\begin{array}{c}\text { Ponto da carga } 10 \\
\text { carga }(\mathrm{cm})\end{array}$ & $\begin{array}{c}\text { distância entre } \\
\text { cargas }(\mathrm{cm})\end{array}$ \\
\hline 0 & 25,0000 & 12,50 & 230 & 200 & 80 & 70 \\
1 & 25,0000 & 12,50 & 280 & 250 & 97,5 & 85 \\
2 & 25,0000 & 12,50 & 330 & 300 & 115 & 100 \\
3 & 25,0000 & 12,50 & 380 & 350 & 130 & 120 \\
4 & 25,0000 & 12,50 & 430 & 400 & 147,5 & 135 \\
5 & 25,0000 & 12,50 & 480 & 450 & 165 & 150 \\
\hline
\end{tabular}

\section{Discussão e Resultados}

A primeira análise é realizada de acordo com a proposição da Tabela 2.3, onde o foco é a mudança de altura de forma que o coeficiente $h / b$ aumente de 2 para 3 .

Para essa análise a Figura 23.1 apresenta os resultados obtidos através da simulação numérica da influência da variação de altura. Lembrando que em todos os itens dessa análise não houve variação no volume de fibras metálicas.

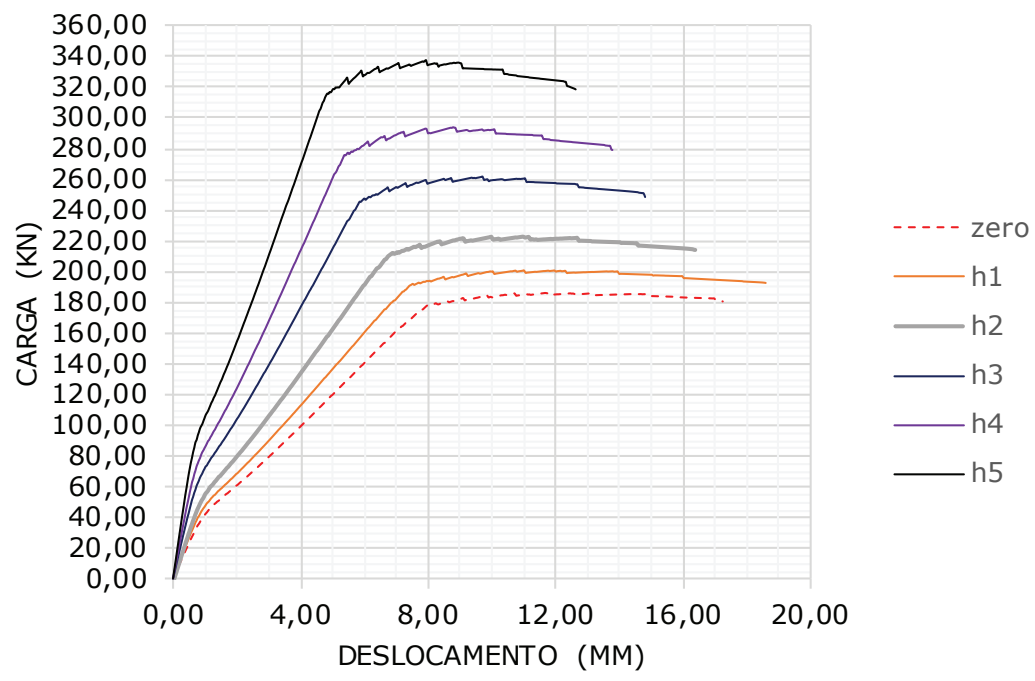

Figura 23.2 Carga versus deslocamento para mudança de altura 
Pode-se afirmar que os ganhos de resistência e de rigidez foram bastante acentuados para incrementos de altura. É possível observar a Figura 23.1 e detectar que o aumento da mesma proporciona um ganho de resistência na peça como um todo, desde seu Estado Limite de Serviço (ELS) até o seu Estado Limite Último (ELU). O aumento da carga última da estrutura para o caso h5 foi elevado em até $81,56 \%$ quando comparado ao teste 0 . A Tabela 3.1 apresenta de forma mais detalhada o ganho total em cada teste realizado e para verificar que o aumento da carga não é linear apresenta-se a Figura 23.2.

Tabela 23.7 Cargas totais para os testes com mudança de altura

\begin{tabular}{ccccc}
\hline Teste & $\begin{array}{c}\text { Carga máxima } \\
(\mathrm{kN})\end{array}$ & $\begin{array}{c}\text { Carga do ELS } \\
(\mathrm{kN})\end{array}$ & $\begin{array}{c}\text { Aumento de } \\
\text { carga em kN }\end{array}$ & $\begin{array}{c}\text { Aumento de carga em } \\
\%\end{array}$ \\
\hline 0 & 186,17 & 74,468 & ---- & ---- \\
1 & 200,422 & 80,1688 & 14,252 & $7,66 \%$ \\
2 & 223,022 & 89,2088 & 36,852 & $19,79 \%$ \\
3 & 262,359 & 104,9436 & 76,189 & $40,92 \%$ \\
4 & 294,479 & 117,7916 & 108,309 & $58,18 \%$ \\
5 & 338,001 & 135,2004 & 151,831 & $81,56 \%$ \\
\hline
\end{tabular}

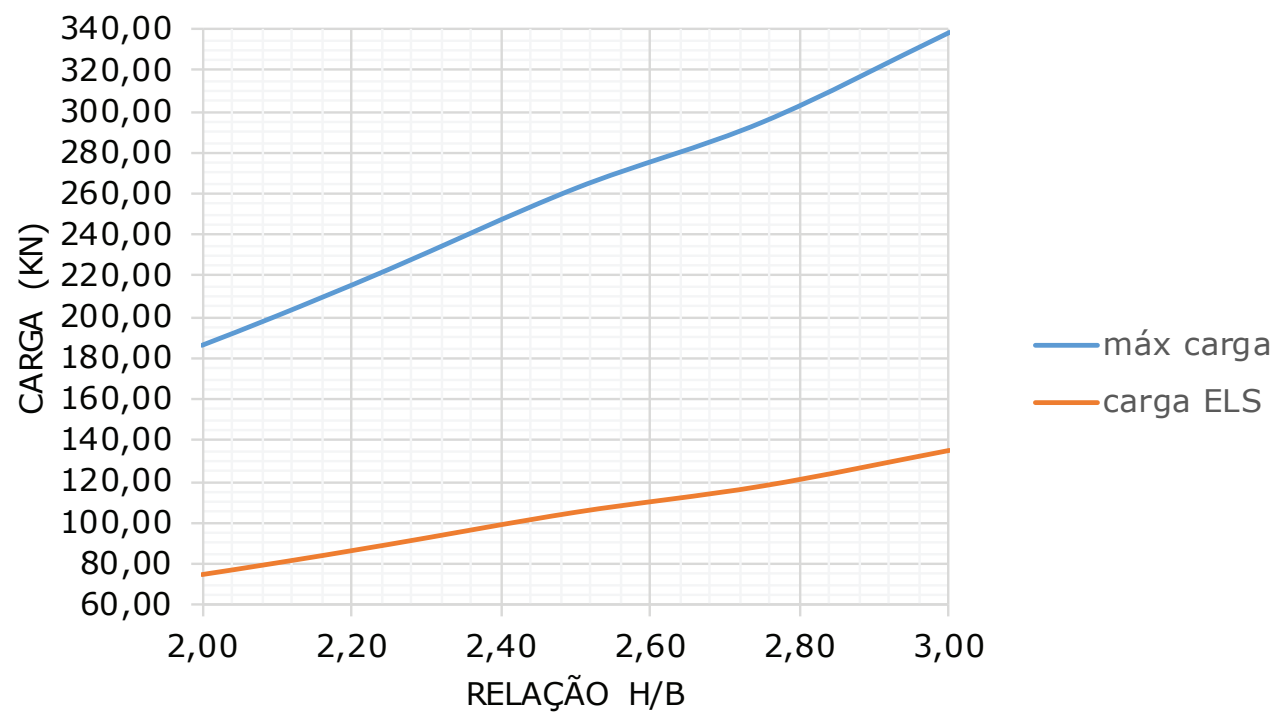

Figura 23.3 Comparativo de cargas para a relação $h / b$

Avaliando o aumento dos deslocamentos da peça, a Tabela 3.2 apresenta a comparação do ganho de carga em função de pontos de medidas de deslocamentos, que nessa Tabela foram cerca de: (a) $4 \mathrm{~mm}$; (b) $8 \mathrm{~mm}$; (c) $12 \mathrm{~mm}$. 
Tabela 23.8 Comparativo carga e deslocamento para 4, 8 e 12 mm aproximadamente

\begin{tabular}{ccccccc}
\hline Teste & $\begin{array}{c}\text { Desloc. 4 } \\
\mathrm{mm}(\mathrm{mm})\end{array}$ & $\begin{array}{c}\text { Carga a } \\
4 \mathrm{~mm} \\
(\mathrm{kN})\end{array}$ & $\begin{array}{c}\text { Desloc. 8 } \\
\mathrm{mm}(\mathrm{mm})\end{array}$ & $\begin{array}{c}\text { Carga a 8 } \\
\mathrm{mm}(\mathrm{kN})\end{array}$ & $\begin{array}{c}\text { Desloc. } \\
12 \mathrm{~mm} \\
(\mathrm{~mm})\end{array}$ & $\begin{array}{c}\text { Carga a } \\
12 \mathrm{~mm} \\
(\mathrm{kN})\end{array}$ \\
\hline 0 & 4,01 & 99,51 & 8,01 & 178,43 & 12,03 & 185,24 \\
1 & 4,01 & 112,80 & 8,01 & 193,43 & 12,00 & 199,73 \\
2 & 4,01 & 134,87 & 8,01 & 217,37 & 12,02 & 221,62 \\
3 & 4,01 & 177,10 & 8,03 & 258,03 & 12,02 & 258,19 \\
4 & 4,01 & 214,35 & 8,03 & 290,78 & 12,03 & 285,81 \\
5 & 4,00 & 269,41 & 8,00 & 334,93 & 12,01 & 324,98 \\
\hline
\end{tabular}

É possível verificar que o carregamento a $4 \mathrm{~mm}, 8 \mathrm{~mm}$ e $12 \mathrm{~mm}$ aumentou consideravelmente visualizando que a peça adquiriu resistência com o aumento da altura.

O próximo teste realizado foi do aumento da largura com os mesmos valores propostos em h/b. A proposição dos testes realizados foi feita na Tabela 2.4. A Figura 23.3 apresenta os resultados gráficos da curva carga versus deslocamento para essa situação.

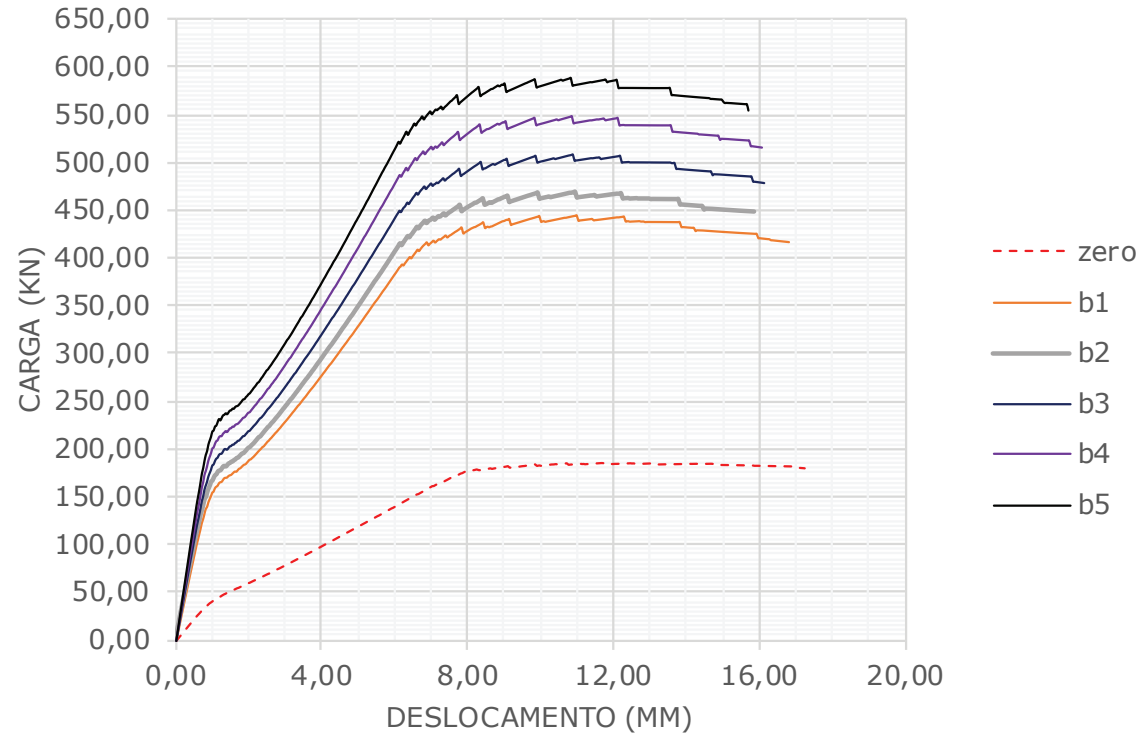

Figura 23.4 Carga versus deslocamento para mudança da largura

É possível perceber que o aumento de largura proporciona resistências superiores ao aumento da altura, porém ressalta-se que isso se deve ao fato que a largura foi praticamente duplicada para os testes realizados e que isso leva a um aumento de quantidade relativa de fibras. Já na Figura 23.4 são comparados os gráficos das Figuras 23.1 e 23.3, onde percebe-se que o ganho de resistência rela- 
tiva de um teste de altura é maior que o ganho de resistência relativa de um teste de largura.

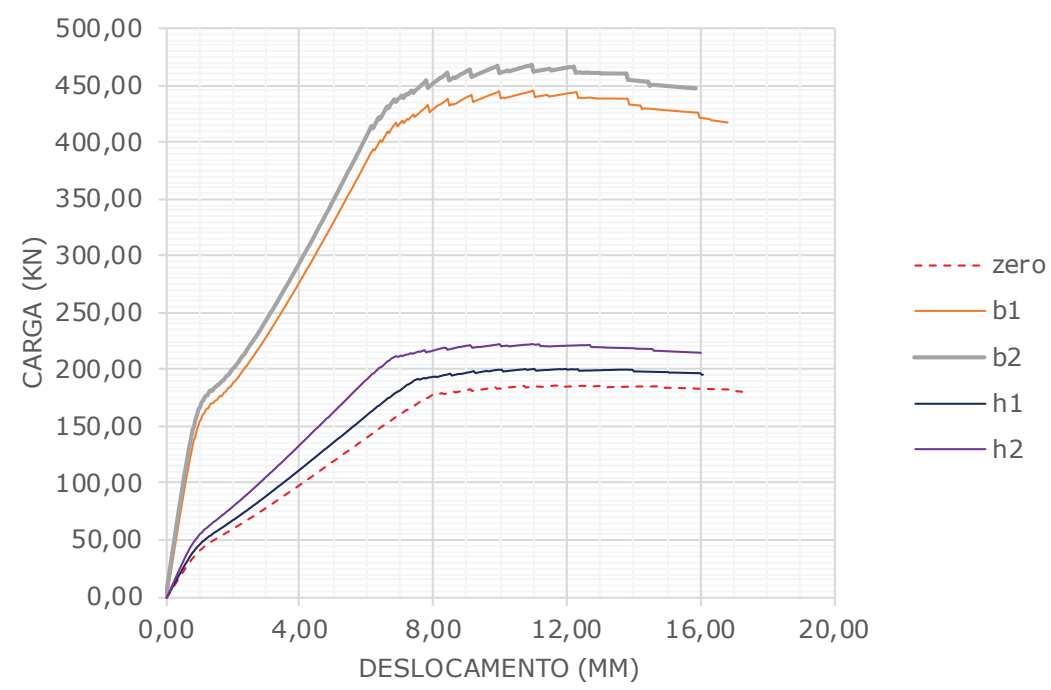

Figura 23.5 Comparação dos valores de aumento da altura e da largura

Analisando de forma mais detalhada os gráficos, o incremento de altura proporciona um ganho mais acentuado de resistência. Para o teste apresentado na Figura 23.4 esse ganho total é de 11,28\% enquanto incrementos de altura proporcionam um aumento de $5,37 \%$ de resistência.

Conforme as análises anteriores a Tabela 23.3 apresenta os valores de aumento de resistência para o incremento de largura.

Tabela 23.9 Cargas totais para os testes com mudança de altura

\begin{tabular}{ccccc}
\hline Teste & $\begin{array}{c}\text { Carga } \\
\text { máxima } \\
(\mathrm{kN})\end{array}$ & $\begin{array}{c}\text { Carga do } \\
\text { ELS }(\mathrm{kN})\end{array}$ & $\begin{array}{c}\text { Aumento } \\
\text { de carga } \\
\text { em kN }\end{array}$ & $\begin{array}{c}\text { Aumento } \\
\text { de carga } \\
\text { em \% }\end{array}$ \\
\hline 0 & 186,17 & 74,468 & --- & --- \\
1 & 444,51 & 177,8032 & 258,338 & $138,76 \%$ \\
2 & 468,37 & 187,348 & 282,2 & $151,58 \%$ \\
3 & 508,19 & 203,2768 & 322,022 & $172,97 \%$ \\
4 & 548,13 & 219,25 & 361,955 & $194,42 \%$ \\
5 & 587,95 & 235,1812 & 401,783 & $215,82 \%$ \\
\hline
\end{tabular}

Esse valor de aumento maior que $100 \%$ se deve ao fato que o valor da largura foi dobrado para o processo iterativo. A Tabela 3.4 apresenta os valores de carga e deslocamento para três momentos da análise. 
Tabela 23.10 Comparativo carga e deslocamento para 4, 8 e 12 mm aproximadamente

\begin{tabular}{ccccccc}
\hline Teste & $\begin{array}{c}\text { Desloc. 4 } \\
\mathrm{mm}(\mathrm{mm})\end{array}$ & $\begin{array}{c}\text { Carga a } \\
4 \mathrm{~mm} \\
(\mathrm{kN})\end{array}$ & $\begin{array}{c}\text { Desloc. 8 } \\
\mathrm{mm}(\mathrm{mm})\end{array}$ & $\begin{array}{c}\text { Carga a 8 } \\
\mathrm{mm}(\mathrm{kN})\end{array}$ & $\begin{array}{c}\text { Desloc. } \\
12 \mathrm{~mm} \\
(\mathrm{~mm})\end{array}$ & $\begin{array}{c}\text { Carga a } \\
12 \mathrm{~mm} \\
(\mathrm{kN})\end{array}$ \\
\hline 0 & 4,01 & 99,51 & 8,01 & 178,43 & 12,03 & 185,24 \\
1 & 4,00 & 277,80 & 8,02 & 429,20 & 12,02 & 442,15 \\
2 & 4,00 & 294,09 & 8,02 & 452,85 & 12,02 & 466,07 \\
3 & 4,00 & 321,27 & 8,03 & 491,85 & 12,03 & 505,91 \\
4 & 4,00 & 348,55 & 8,03 & 531,27 & 12,03 & 545,83 \\
5 & 4,01 & 375,48 & 8,03 & 570,59 & 12,03 & 585,64 \\
\hline
\end{tabular}

O próximo teste realizado trata da redução da área de aço na seção transversal considerando perdas de 10\%, 20\%, 30\%, 40\% e 50\% conforme Tabela 2.5 . A apresentação dos resultados é feita na Figura 23.5.

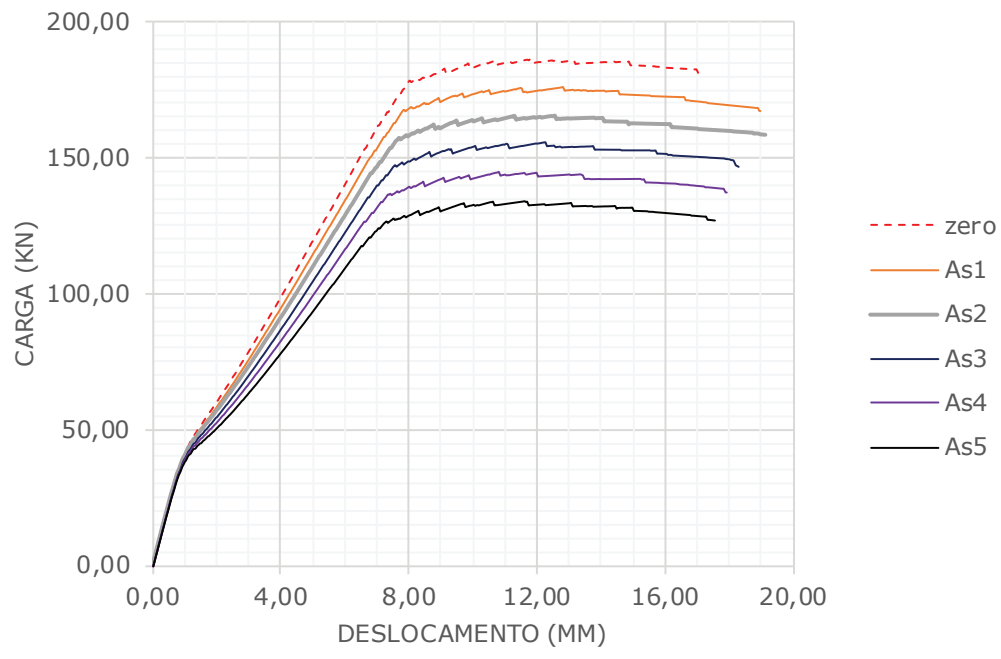

Figura 23.6 Comparação dos valores de redução da área de aço

Conforme já era esperado, reduções de armadura comprometem a resistência da peça. Para visualizar a queda na resistência da peça as Tabelas 3.5 e 3.6 são apresentadas logo abaixo:

Tabela 23.1 1 Redução da carga para situaaçōes das armaduras

\begin{tabular}{ccccc}
\hline Teste & $\begin{array}{c}\text { Carga } \\
\text { máxima } \\
(\mathrm{kN})\end{array}$ & $\begin{array}{c}\text { Carga do } \\
\text { ELS }(\mathrm{kN})\end{array}$ & $\begin{array}{c}\text { Redução } \\
\text { de carga } \\
\text { em kN }\end{array}$ & $\begin{array}{c}\text { Redução } \\
\text { de carga } \\
\text { em \% }\end{array}$ \\
\hline 0 & 186,17 & 74,468 & --- & ---- \\
1 & 176,34 & 70,5376 & 9,826 & $5,28 \%$ \\
2 & 165,71 & 66,2852 & 20,457 & $10,99 \%$ \\
3 & 155,75 & 62,3004 & 30,419 & $16,34 \%$ \\
4 & 144,96 & 57,9844 & 41,209 & $22,14 \%$ \\
5 & 134,22 & 53,6872 & 51,952 & $27,91 \%$ \\
\hline
\end{tabular}


Tabela 23.12 Comparativo carga e deslocamento para 4, 8 e 12 mm aproximadamente

\begin{tabular}{ccccccc}
\hline Teste & $\begin{array}{c}\text { Desloc. 4 } \\
\mathrm{mm}(\mathrm{mm})\end{array}$ & $\begin{array}{c}\text { Carga a } \\
4 \mathrm{~mm} \\
(\mathrm{kN})\end{array}$ & $\begin{array}{c}\text { Desloc. 8 } \\
\mathrm{mm}(\mathrm{mm})\end{array}$ & $\begin{array}{c}\text { Carga a 8 } \\
\mathrm{mm}(\mathrm{kN})\end{array}$ & $\begin{array}{c}\text { Desloc. } \\
12 \mathrm{~mm} \\
(\mathrm{~mm})\end{array}$ & $\begin{array}{c}\text { Carga a } \\
12 \mathrm{~mm} \\
(\mathrm{kN})\end{array}$ \\
\hline 0 & 4,013 & 99,509 & 8,01 & 178,43 & 12,03 & 185,24 \\
1 & 4,02 & 95,60 & 8,02 & 168,76 & 12,01 & 175,06 \\
2 & 4,02 & 91,65 & 8,00 & 158,40 & 12,02 & 165,16 \\
3 & 4,02 & 87,61 & 8,01 & 148,68 & 12,03 & 155,35 \\
4 & 4,02 & 83,31 & 8,01 & 139,58 & 12,00 & 144,67 \\
5 & 4,02 & 78,91 & 8,03 & 129,15 & 12,02 & 132,97 \\
\hline
\end{tabular}

Observando a Tabela 3.5 é possível verificar que, mesmo para situações de $50 \%$ de redução na armadura longitudinal, a carga não sofreu alterações maiores que $20 \%$ em redução da carga máxima. Esse fato pode ser devido a presença de fibras metálicas na composição do concreto armado. Para situações sem a presença da fibra metálica esses valores seriam mais acentuados conforme observações do trabalho de Schmaltz et al. (2011).

Finalizando a composição de resultados do problema, variou-se o vão de forma a obter os valores de carga versus deslocamento. Os valores dessas simulações seguem na Tabela 3.7. Maiores detalhes dessa simulação são apresentados nas Figuras 23.6 e Tabela 23.8.

Para o aumento de vão é possível verificar que a carga decresce em valores na faixa de $21,23 \%$ a $62,93 \%$. Quando comparado aos valores de armadura esse decréscimo é bem mais acentuado devido ao aumento do valor de momento fletor na peça, enquanto o problema de armadura mantém o momento na seção transversal.

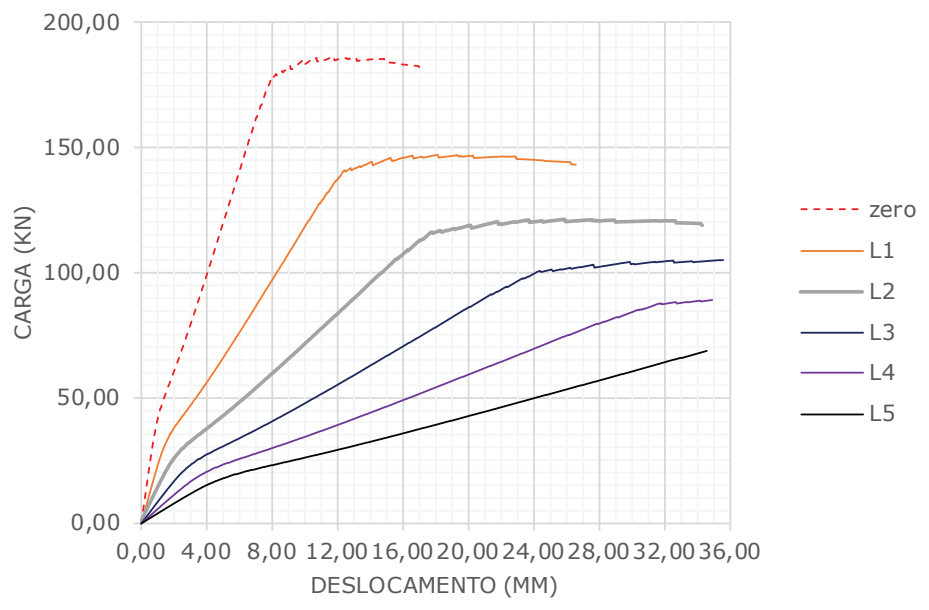

Figura 23.7 Comparação dos valores para aumento do vão 
Tabela 23.13 Redução da carga para situações do vão

\begin{tabular}{ccccc}
\hline Teste & $\begin{array}{c}\text { Carga } \\
\text { máxima } \\
(\mathrm{kN})\end{array}$ & $\begin{array}{c}\text { Carga do } \\
\text { ELS }(\mathrm{kN})\end{array}$ & $\begin{array}{c}\text { Redução } \\
\text { de carga } \\
\text { em kN }\end{array}$ & $\begin{array}{c}\text { Redução } \\
\text { de carga } \\
\text { em \% }\end{array}$ \\
\hline 0 & 186,17 & 74,468 & ---- & ---- \\
1 & 146,655 & 58,662 & 39,515 & $21,23 \%$ \\
2 & 121,027 & 48,4108 & 65,143 & $34,99 \%$ \\
3 & 105,202 & 42,0808 & 80,968 & $43,49 \%$ \\
4 & 88,8438 & 35,53752 & 97,3262 & $52,28 \%$ \\
5 & 69,0139 & 27,60556 & 117,1561 & $62,93 \%$ \\
\hline
\end{tabular}

Verificando os valores de carga versus deslocamento do problema com a mudança de vão apresenta-se a Tabela 3.8.

Tabela 23.14 Comparativo carga e deslocamento para 4, 8 e 12 mm aproximadamente

\begin{tabular}{ccccccc}
\hline Teste & $\begin{array}{c}\text { Desloc. 4 } \\
\mathrm{mm}(\mathrm{mm})\end{array}$ & $\begin{array}{c}\text { Carga a } \\
4 \mathrm{~mm} \\
(\mathrm{kN})\end{array}$ & $\begin{array}{c}\text { Desloc. 8 } \\
\mathrm{mm}(\mathrm{mm})\end{array}$ & $\begin{array}{c}\text { Carga a 8 } \\
\mathrm{mm}(\mathrm{kN})\end{array}$ & $\begin{array}{c}\text { Desloc. } \\
12 \mathrm{~mm} \\
(\mathrm{~mm})\end{array}$ & $\begin{array}{c}\text { Carga a } \\
12 \mathrm{~mm} \\
(\mathrm{kN})\end{array}$ \\
\hline 0 & 4,013 & 99,509 & 8,01 & 178,43 & 12,03 & 185,24 \\
1 & 4,00 & 56,06 & 8,01 & 97,02 & 12,01 & 136,98 \\
2 & 4,01 & 37,27 & 8,02 & 59,32 & 12,02 & 83,20 \\
3 & 4,00 & 27,52 & 8,01 & 40,80 & 12,00 & 55,36 \\
4 & 4,00 & 20,57 & 8,02 & 30,03 & 12,01 & 39,18 \\
5 & 4,01 & 15,35 & 8,02 & 23,32 & 12,03 & 29,50 \\
\hline
\end{tabular}

\section{Conclusões ou considerações finais}

Os resultados elencados no item 3 mostram que altura e vão talvez sejam as propriedades mais influentes no quesito de análise da deformabilidade de peças fissuradas. Os estudos apontaram que para aumento da altura da seção transversal, observam-se aumentos sensíveis de aproximadamente $80,00 \%$ para as simulações indicadas. Já para o vão, a mudança é sensível no sentido de redução da carga máxima da peça. A situação 4 e 5 apontam uma baixa de mais de 50,00\%.

Provavelmente esses aumentos de carga foram incrementados pela presença da fibra metálica na matriz de concreto, visto que a concentração utilizada para essas simulações foi de $2,00 \%$ de fibras.

Outro fato importante se dá na análise das reduções de armadura longitudinal, onde foi possível verificar que em reduções expressivas como de 50,00\% a menos de armadura a queda de resistência da peça foi de apenas de aproximadamente $27,00 \%$. Portanto, observa-se que a presença de fibras na matriz cimentícia reduz o nível de deslocamento na peça, mesmo que haja uma redução na armadura. Fato o qual é explicado pelo efeito de grampeamento das fissuras. 
As sugestões para trabalhos futuros são verificar experimentalmente a influência dos parâmetros geométricos na deformabilidade da peça fissurada, além da utilização de outros tipos de concreto e concentração de fibras na mistura, permitindo assim criar um campo de resultados que permitam conclusões mais abrangentes sobre tal tema, além de contribuir para uma discussão sobre o cálculo de deslocamentos em peças de concreto reforçado com fibras.

\section{Referências}

ABNT - ASSOCIAÇÃO BRASILEIRA DE NORMAS TÉCNICAS. NBR 15530: Fibras de aço para concreto - Especificação. Rio de Janeiro, 2007, p. 7.

ABNT - ASSOCIAÇÃO BRASILEIRA DE NORMAS TÉCNICAS. NBR 8890: Tubo de concreto de seção circular para águas pluviais e esgotos sanitários - Requisitos e métodos de ensaios. Rio de Janeiro, 2007, 30 p.

LA BORDERIE, C. Phenomenes unilateraux dans un materiau endommageable: modelisation et application a l'analyse de structures en beton. $140 \mathrm{f}$. Tese (Doutorado em Engenharia) - Universidade de Paris, Paris, 1991.

LOPES, M. M. Substituição parcial de armadura de flexão por fibras de aço em vigas de concreto. 2005. 155 f. Dissertação (Mestrado em Engenharia Civil) Universidade Federal do Rio de Janeiro, Rio de Janeiro, 2005.

PASA, V. F. Análise do comportamento de estruturas de concreto reforçado com fibras de aço via método dos elementos finitos. 2007. 130 f. Dissertação (Mestrado em Engenharia de Estruturas) - Universidade Federal do Rio Grande do Sul, Porto Alegre, 2007.

PEREIRA JUNIOR, W. M. Análise numérica de estruturas de concreto com fibras utilizando mecânica do dano. 2014. 157 f. Dissertação (Mestrado em Mecânica das Estruturas) - Escola de Engenharia de Engenharia Civil e Ambiental, Universidade Federal de Goiás, Goiânia, 2014.

PEREIRA JUNIOR, W. M.; PITUBA, J. J. C.; ARAÚJO, D. L. (2016). Análise numérica de vigas de concreto com fibras de aço utilizando mecânica do dano. Revista Ibracon de Estruturas e Materiais, v. 9, pp 153-191.

PITUBA, J. J. C. Sobre a formulação de um modelo de dano para concreto. 2003. $151 \mathrm{f}$. Tese (Doutorado em Engenharia de Estruturas) - Escola de Engenharia de São Carlos, Universidade de São Paulo, São Paulo, 2003. 
PITUBA, J. J. C.; FERNANDES, G. R. (2011). An Anisotropic Damage Model for Concrete. Journal of Engineering Mechanics, v. 137, pp 610-624.

SCOARIS, M. R. Concretos Reforçados com Fibras: Avaliação das Propriedades Mecânicas Através do Método da Maturidade. 2005. 260 f. Dissertação (Mestrado em Engenharia de Estruturas) - Faculdade de Engenharia de Ilha Solteira, Universidade Estadual Paulista, Ilha Solteira, 2005.

SCHMALTZ, F. A. V.; PEREIRA JUNIOR, W. M.; BATISTA, C. M. P.; SALES, M. W. R. (2015). Efeito paramétrico da armadura em pórticos de concreto armado utilizando mecânica do dano. Revista eletrônica de Educação da Faculdade Araguaia, v. 8, pp 224-245. 